\title{
Pretreatment with Shenxiong Drop Pill induces AQP4- mediated neuroprotective effect on middle cerebral artery occlusion in rats
}

\author{
Shuo-guo Jin ${ }^{1}$, Ji-li Deng ${ }^{2}$, Ze-ran Chen ${ }^{3}$, Fang Yang ${ }^{1}$, Mei-jun Liu1, Hong-hui \\ Sun ${ }^{1}$, Ning-jing Ran ${ }^{1}$, Li Zhang ${ }^{4}$, Dong-dong Yang ${ }^{1}$, Wei-yin Chen ${ }^{1 *}$ \\ ${ }^{1}$ Neurology Department, Hospital of Chengdu University of Traditional Chinese Medicine, Chengdu 610072, Sichuan Province, \\ ${ }^{2}$ Department of Traditional Chinese Medical, Xinqiao Hospital, Third Military Medical University, Chongqing 400037, ${ }^{3}$ School of \\ Medical and Life Sciences, Chengdu University of Traditional Chinese Medicine, Chengdu 610041. Sichuan Province, \\ ${ }^{4}$ Department of Internal Medicine No. 1, Chengdu Shuangliu District Chinese Medicine Hospital, Chengdu, 610200, Sichuan \\ Province, PR China
}

*For correspondence: Email: chenwydoctor@sina.com, yangdongdong2@126.com; Tel: +86-028-87783362, +86-02887783362

\begin{abstract}
Purpose: To investigate the neuroprotective effect of Shenxiong Drop Pill (SXDP) pretreatment on rats with middle cerebral artery occlusion (MCAO) in rats, and the mechanism involved.

Methods: Ninety-nine SD rats were randomly assigned to 4 groups: control group, MCAO group, shamoperated group and SXDP group. The MCAO model was established via thread occlusion. Rats in the SXDP group was administered SXDP 7 days before induction of MCAO. Neurological deficit score (NDS) was determined using Bederson's neurological behavioral scoring method, while cerebral infarction volume was measured using TTC staining. Integrated optical density (IOD) of Nissl Body was evaluated via Nissl staining. Brain water content was measured using dry-wet method. The expression level of AQP4 in brain tissues was determined using immunocytochemistry.

Results: The SXDP treatment resulted in significant reduction in NDS, marked improvement in IOD of Nissl Body, and significant reductions in cerebral infarction volume, brain water content, and expression level of AQP4, relative to control ( $p<0.05)$.

Conclusion: These results suggest that SXDP pretreatment exerts neuroprotective effect against cerebral ischemia in rats by decreasing in cerebral edema through a mechanism involving downregulation of the expression of AQP4.
\end{abstract}

Keywords: Middle cerebral artery occlusion, Cerebral ischemia, Aquaporins-4, Cerebral edema, Neuroprotection

This is an Open Access article that uses a fund-ing model which does not charge readers or their institutions for access and distributed under the terms of the Creative Commons Attribution License (http://creativecommons.org/licenses/by/4.0) and the Budapest Open Access Initiative (http://www.budapestopenaccessinitiative.org/read), which permit unrestricted use, distribution, and reproduction in any medium, provided the original work is properly credited.

Tropical Journal of Pharmaceutical Research is indexed by Science Citation Index (SciSearch), Scopus, International Pharmaceutical Abstract, Chemical Abstracts, Embase, Index Copernicus, EBSCO, African Index Medicus, JournalSeek, Journal Citation Reports/Science Edition, Directory of Open Access Journals (DOAJ), African Journal Online, Bioline International, Open-J-Gate and Pharmacy Abstracts

\section{INTRODUCTION}

In recent years, stroke has become a leading cause of death in China [1]. The burden associated with treating and caring for stroke patients has increased over the past 30 years [2]. Individuals who experience ischemic stroke, which accounts for about $70-80 \%$ of all strokes, might be permanently paralyzed on one side of 
the body, and/or may lose the ability to speak intelligibly. Thus, there is an important medical need for effective treatment strategies for ischemic stroke.

Shenxiong Drop Pill (SXDP) is a compound Chinese herbal preparation composed of extracts from different plants i.e. Salvia miltiorrhiza Bge, Ligusticum Chuanxiong Hort., and Astragali Radix. The bioactive components are Astragalus saponins, total salvianolic acid, and volatile oils from Ligusticum chuanxiong. In this study, experiments were conducted to investigate the effect of SXDP pretreatment on acute ischemic stroke in rats, and the underlying mechanism.

In previous studies, it was found that SXDP had a positive effect on acute ischemic stroke which was associated with increases in the expression level of Nissl bodies [3], regulation of the balance between t-PA and PAl-1 in the plasma of rats with middle cerebral artery occlusion (MCAO) [6], increased expression of proteins related to the growth of neurites (GAP-43 and SYN), increased proliferation and differentiation of endogenous neural stem cells, and promotion of neuroplasticity after ischemia [5]. Moreover, SXDP decreased inflammatory reaction and nerve cell apoptosis in rats at the early stage of cerebral infarction by downregulating the expressions of CD11/CDI8, ICAM-1 mRNA, TNF-amRNA, NF-kB, and caspase $3[4,7]$. However, not much is known about the effect of SXDP pretreatment on acute ischemic stroke, and the underlying mechanism. Therefore, this study was carried out to investigate the neuroprotective effect of SXDP pretreatment on MCAO in rats, and its possible association with the brain expression level of aquaporin-4 (AQP4).

Research on stroke is focused mainly on the endogenous neuroprotective mechanisms by which the brain protects itself against ischemia and promotes recovery from ischemic damage. Previous studies based on post-MCAO administration of SXDP have revealed that it has some neuroprotective effects. In addition, the use of SXDP pretreatment has achieved some good results in stroke, but there are limited studies regarding SXDP pretreatment in relation to MCAO. Recombinant AQP4 participates in the formation and elimination of edema, indicating that AQP4 regulators may provide new avenues for the clinical treatment of cerebral edema [8]. In the present study, the effect of SXDP pretreatment on AQP4 levels in the brains of MCAO rats was investigated.

\section{EXPERIMENTAL}

\section{Animals}

A total of 99 male adult Sprague-Dawley (SD) rats weighing 250 - $300 \mathrm{~g}$ were purchased from the Experimental Animal Center of Chengdu University of Traditional Chinese Medicine (TCM), and were raised under standard conditions before and after surgery in the Time Biology Laboratory of Chengdu University of TCM. The room temperature was kept at 23-25 ${ }^{\circ} \mathrm{C}$. All rats were allowed free access to feed and water, and were fed for 7 days to adapt them to the laboratory environment. The rats were randomly assigned to 4 groups: control, MCAO, sham-operated, and SXDP groups. This study was approved by the Laboratory Animal Welfare and Ethics Committee of Chengdu University of TCM, China (approval no. 2016-13). All experimental procedures involving rats were undertaken in accordance with the principles of Laboratory Animal Care and the Guide for the Care and Use of Laboratory Animals, published by the National Science Council, China. Fresh feed and water were provided ad libitum to all animal groups.

\section{Drugs and reagents}

Shenxiong Drop Pill (SXDP) was produced by the Pharmacy School of Chengdu University of TCM (Patent number: ZL200910262686, China). The dose of SXDP was $10 \mathrm{~g} / \mathrm{kg}$. It was prepared as a suspension containing $5.5 \mathrm{~g} / \mathrm{mL}$. The rats received SXDP via gavage administration of the suspension in succession before induction of MACO. Horseradish peroxidase-conjugated AffiniPure Goat Anti-Rabbit IgG (ZB-2301), SPlink Detection kit (rat/rabbit) (SP-9000), and diaminobenzidine (DAB) staining kit (ZLI-9019) were purchased from Beijing Zhongshanjinqiao Biotechnology Co. (China), while AQP4 antibody (SC-327393, mouse anti-rat lgG) was purchased from Santa Cruz Biotechnology (USA).

\section{Drug administration}

The SXDP group received intragastric administration of SXDP suspension $(10 \mathrm{mg} / \mathrm{kg}$, once a day) for 7 days before MCAO surgery. At the same time, the control, sham-operated, and MCAO groups were administered equivalent volumes of normal saline daily in place of SXDP.

\section{Establishment of MCAO rat model}

The rats were subjected to MCAO according to the MCA bolt line blocking method of Zea Longa [9]. Preoperative anesthesia and vascular 
segregation were applied, in which the carotid artery, internal carotid artery, and external carotid artery were exposed, separated, and blocked via intraluminal suture. The indoor temperature was maintained at $24-25^{\circ} \mathrm{C}$ during the surgery. The rats were anesthetized with 2 $\%$ sodium pentobarbital (F200209150; Shanghai Chemical Reagent Company, China). During the surgery, the rectal temperature was recorded and maintained from 36.5 to $37.5{ }^{\circ} \mathrm{C}$ via a thermostatically- controlled heating pad.

The rats were placed in the supine position, and the external carotid artery and its branches, occipital artery, and superior thyroid artery were dissociated, and the branches were ligated. Then, the ipsilateral external carotid artery (ECA) was isolated and ligated at approximately $0.8 \mathrm{~cm}$ from the ECA. A V-shaped incision with a diameter of about $2 \mathrm{~mm}$ was made between the ECA ligation and the bifurcation at the proximal end of the common carotid artery (CCA). A fishing line was gently inserted into the CCA from the incision, and the artery clamp was released. The bifurcation of the internal and external carotid arteries entered the internal carotid artery (ICA), and the fishing line was slowly advanced into the cranial direction of the ICA. When the insertion depth was about $18.5 \pm$ $0.5 \mathrm{~mm}$, and upon feeling micro-resistance, the end of the fishing line arrived at the anterior cerebral artery along the beginning of the middle cerebral artery (MCA). By this method, the blood flow to the left MCA was obstructed. The ICA was ligated so as to fix the fishing line, bleeding was stopped, and the incision was sutured layerby-layer. The stump residue was $1 \mathrm{~cm}$ longer than the skin and was marked. A total of 5 animals died in the MCAO and SXDP groups. These comprised 1 anesthesia accident, 1 unqualified score, 1 death from incision infection, and 2 deaths from subarachnoid hemorrhage. The total success of this procedure was $70.5 \%$ (excluding the normal and sham groups).

\section{Neurologic deficit score test}

In accordance with the Bederson method [10], neurological deficits were scored and recorded $24 \mathrm{~h}$ after establishment of the MCAO model. The scoring criteria were as follows: score $0=$ no symptoms of nerve injury; score $1=$ moderate forelimb flexion; score 2 = circling to the paralyzed side during walking; score $3=$ falling to the contralateral side of the lesion, and score 4 = inability to walk spontaneously. The people who conducted the tests and analyses were blind to the various treatments.

\section{Sample processing}

After establishing the neurologic deficit score, all rats were euthanized via intraperitoneal injection of $2 \%$ pentobarbital sodium $24 \mathrm{~h}$ after the MCAO was established, and the brains of rats in each group were isolated. The brain samples were divided into three portions: one portion was used for determination of water content, while another portion was used for measurement of cerebral infarction volume. The third brain portion was fixed in $4 \%$ paraformaldehyde, embedded in paraffin, and sliced for histopathological and immunohistochemical staining.

\section{Determination of cerebral infarction volume}

Triphenyltetrazolium chloride (TTC) staining was used to measure cerebral infarction volume as described previously. The rat brain was frozen at $-20{ }^{\circ} \mathrm{C}$ for $15 \mathrm{~min}$. and sectioned into 2-mm slices which were treated with $2 \%$ TTC solution for $20 \mathrm{~min}$ at $37^{\circ} \mathrm{C}$, and with $4 \%$ paraformaldehyde for $24 \mathrm{~h}$. The principle of this test is that TTC reacts with intact mitochondrial respiratory enzymes to generate a bright red color that contrasts with the pale color of the infarction. The digitized images of the TTCstained brain slice were analyzed with Image-pro Plus 6 software.

\section{Nissl staining of rat brain sections}

Brain sections were used for Nissl staining with cresyl violet. After the images were taken using Olympus image system (IX-B50, Olympus Co. Ltd., Japan), Image-pro Plus 6 software was used for semi-quantitative analysis of the Nissl staining results at a magnification of 200 . The integrated optical density values of 5 samples expressing the strongest visual field were considered as measurement values.

\section{Determination of brain water content}

Dry-wet weight method was used to determine the water content of the brain samples. The procedure involved measuring the wet weight of the brain immediately it was isolated from the cerebral cortex tissue specimens, drying the sample at $110{ }^{\circ} \mathrm{C}$, and weighing the dry weight $24 \mathrm{~h}$ later. The brain water content was calculated as percentage of wet weight.

\section{Immunohistochemistry of AQP-4}

In the ischemic cortex (within $4 \mathrm{~mm}$ posterior to the optic chiasm), coronal paraffin sections were 
sliced to a thickness of 8 um. The level of AQP4 was measured via immunohistochemistry, while the integrated optical density (IOD) was measured using an Image-Pro Plus image analysis system (Media Cybernetics, USA).

The slices were dewaxed for $15 \mathrm{~min}$, rinsed thrice in distilled water, and immersed in $0.01 \mathrm{M}$ citrate buffer $(\mathrm{pH} 6.0)$. Ten slices were then heated to boiling (with the power cut off every 5$10 \mathrm{~min}$ ) and rinsed with $0.02 \mathrm{M}$ citrate buffer once or twice. Thereafter, the tissues were incubated with normal rabbit blocking solution for 20 min at room temperature, and with an AQP4 antibody at $37^{\circ} \mathrm{C}$ for $1 \mathrm{~h}$, followed by another $0.02 \mathrm{M}$ PBS washing thrice for $2 \mathrm{~min}$. Then, the tissues were treated with biotinylated goat antirabbit IgG at $37^{\circ} \mathrm{C}$ for $20 \mathrm{~min}$, rinsed thrice with $0.02 \mathrm{M}$ PBS for $2 \mathrm{~min}$, incubated with SABC reagent at $37{ }^{\circ} \mathrm{C}$ for $20 \mathrm{~min}$, and rinsed four times with $0.02 \mathrm{M}$ PBS for $5 \mathrm{~min}$. The samples were then stained with $\mathrm{DAB}$, counterstained with hematoxylin, dehydrated, permeabilized, mounted, and observed under a light microscope.

\section{Statistical analysis}

The data were analyzed using Statistical Package for the Social Sciences 17.0 software (SPSS, Chicago, IL, USA), and are presented as mean \pm standard deviation (SD). One-way ANOVA was used for determination of the significant differences among the groups. Values of $p<0.05$ were considered statistically significant.

\section{RESULTS}

\section{SXDP pretreatment attenuated acute ischemic deficits}

No obvious neurological impairment was observed in the control and sham groups (Figure 1). A statistically significant increase in neurological deficit score (NDS) was observed in the model group, when compared with that of the control group $(p<0.01)$. The NDS of the SXDP group was lower than that of the MCAO group $(p<0.05)$, implying that SXDP had a mitigating effect on nerve injury. These results (Figure 1) suggest that pretreatment with SXDP had a neuroprotective effect on cerebral ischemia.

\section{SXDP pretreatment increased expression of Nissl body}

As is shown in Figure $2 \mathrm{~A}$, relative to the model group, there were more cell layers in the cytoplasm of neurons, which were arranged closely in the control and the sham operated groups. The cell morphology was intact, and dark blue-stained, massive or granular Nissl bodies were visible. In the model group, part or all of the Nissl bodies in neurons were diffuse or completely absent. In the SXDP group, the NissI bodies were partially diffuse or absent in the cytoplasm of the neurons, but there was a significantly higher number of Nissl bodies than in the model group.

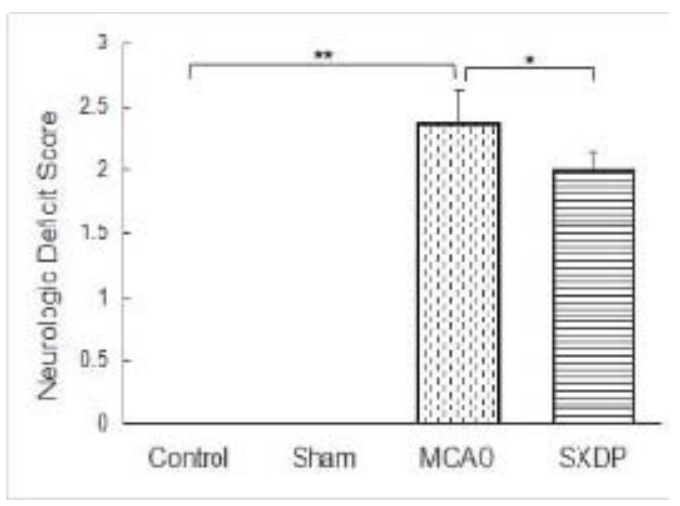

Figure 1: Effect of SXDP pretreatment on neurological deficits score. Results are expressed as mean \pm SD. ${ }^{* *} P<0.01$, versus control group; ${ }^{*} p<$ 0.05 , versus model group $(n=6)$

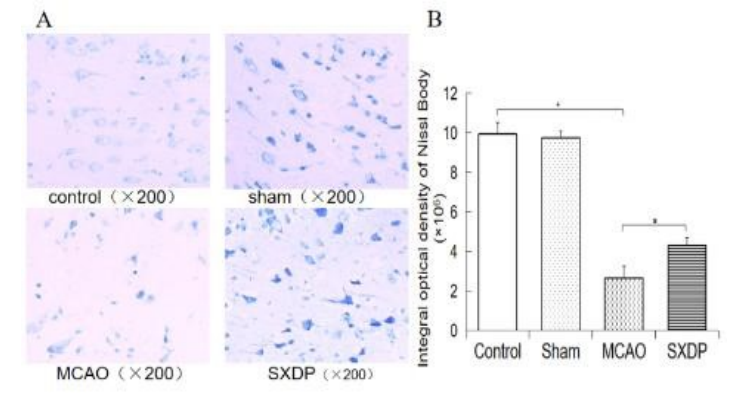

Figure 2: Effect of SXDP pretreatment on the population of Nissl bodies in MCAO rats. Results are expressed as mean $\pm \mathrm{SD}$. ${ }^{* \star} P<0.05$, versus control group; ${ }^{*} p<0.05$, versus model group $(\mathrm{n}=6)$

\section{SXDP pretreatment reduced the area of cerebral infarction}

Cerebral samples of rats in the control and sham-operated groups did not show any evidence of infarction. Compared with the control group, there were significant cerebral infarction lesions in the model group $(p<0.01)$. However, infarct volume in SXDP group was decreased significantly when compared with that of the model group $(p<0.05)$. Thus, SXDP reduced the size of cerebral infarction.

Trop J Pharm Res, August 2020; 19(8): 1718 


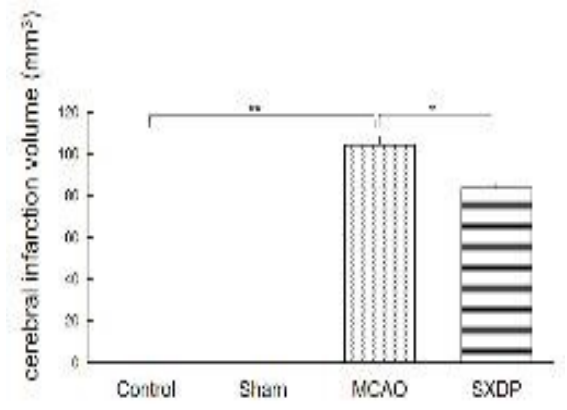

Figure 3: Effect of SXDP pretreatment on cerebral infarction volume of each group. Results are expressed as mean $\pm \mathrm{SD}$. $P<0.01$, versus control group; $p<0.05$, versus model group. (There were 6 rats in the SXDP group, and 7 rats in each of the other three groups

\section{SXDP pretreatment lowered the water content of brain tissues}

The brain water content of each group is shown in Figure 4. There was higher brain water content in the MCAO group than in the shamoperated group $(p<0.01)$. However, SXDP pretreatment resulted in lower brain water content than that in the brain tissues of the MCAO group $(p<0.05)$.

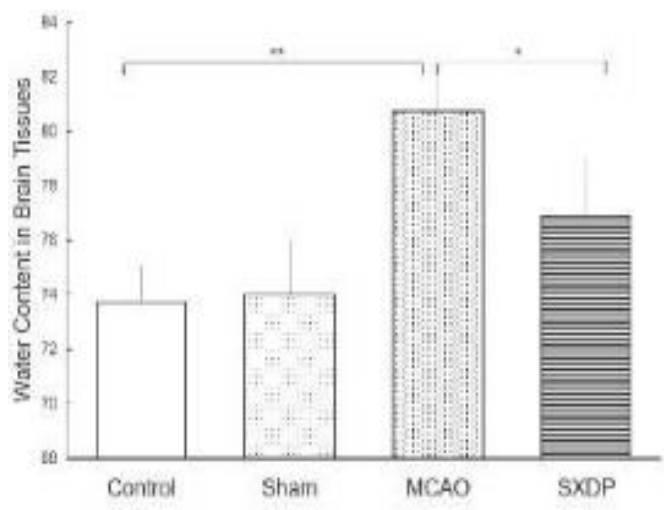

Figure 4: Effect of SXDP pretreatment on brain water content of MCAO rats. Data are expressed as mean \pm SD; $p<0.01$, versus control group; $p<0.05$, versus model group $(n=6)$

\section{SXDP pretreatment reduced the expression level of AQP-4 in brain tissues}

The results of immunohistochemical staining for AQP-4 in the rat brain tissues are shown in Figure 5. With immunohistochemical staining, the AQP4 in the cytoplasm appeared as brownish-yellow spots. It was observed that expression level of AQP-4 in brain tissues was increased in MCAO group, when compared with the control group. However, SXDP pretreatment brought down the level of AQP-4. The IOD representing AQP-4 in the SXDP group was lower than that of the MCAO group $(p<0.01)$.

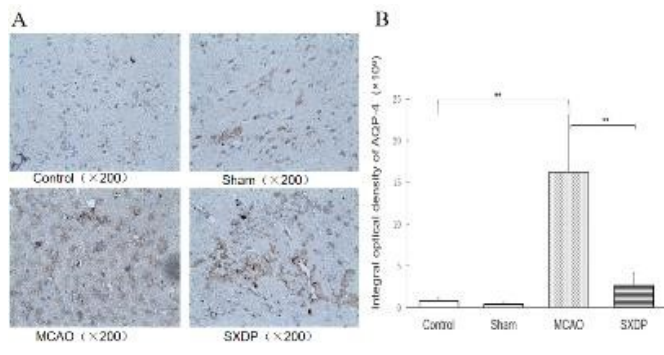

Figure 5: Effect of SXDP pretreatment on the expression level AQP-4 in brain tissues of each group of rats. Results are expressed as mean \pm SD; $p<$ 0.01 , versus control group; $p<0.01$, versus model group $(n=6)$

\section{DISCUSSION}

Cerebral edema is a pathological reaction in response to different kinds of brain damage. In recent years, research on the mechanism of cerebral edema has focused on aquaporins (AQPs) which are a specific family of proteins that function as highly selective water channels. They play an important role in the regulation of fluid metabolism in the body. In the presence of these channels, a considerable amount of water can be transported over a short period of time because of the high-water permeability coefficient of AQPs. This study investigated the effect of pretreatment of SXDP, a herbal compound that can benefit qi and activate the blood to resolve blood stasis, on rats with MCAO. Preliminary studies have showed that SXDP decreased the size of the ischemic region of rats with $\mathrm{MACO}$, increased the expression level of Nissl bodies, regulated balance between t-PA and PAI-1 in the plasma [6], increased the expression of proteins related growth of neuritis (GAP-43, SYN), promoted the proliferation and differentiation of endogenous neural stem cells, and enhanced the occurrence of neuroplasticity after ischemia [5], indicating that it might act on multiple pathways and/or targets [3]. Moreover, SXDP inhibited inflammatory reactions and suppressed nerve cell apoptosis at an early stage of cerebral infarction in rats by downregulating the expressions of CD11/CDI8, ICAM-1mRNA, TNF-amRNA, NF-kB, and Caspase $3[4,7]$.

In the present study, it was found that pretreatment with SXDP for 7 days attenuated the acute neurological deficits and water content in the brains of the rats, reduced infarct volume, increased expression of Nissl Body, and exerted protective effects on cerebral 
ischemia injury in rats. Moreover, it was found that SXDP pretreatment significantly attenuated the expression of AQP4. During ischemic stroke, one important aggravating factor is the presence of edema. Early edema formation significantly contributes to infarct formation, even in non-lacunar ischemic stroke. Brain edema is an independent predictor of a poor outcome, making edema a promising therapeutic target. Following the discovery of AQPs, it has become clear that the various mammalian AQPs play critical physiological roles in the regulation of water and ion balance in multiple tissues. The glial membrane channel AQP4, previously classified as the mercuryinsensitive water channel by Verkman's group, is the most abundant water channel in the brain. It is predominantly localized in astrocyte perivascular end-feet and glial limiting membranes, at the border between the brain parenchyma and subarachnoid CSF, and beneath the ependyma bordering the brain parenchyma and ventricular CSF.

Aquaporin (AQP4) has been shown to mediate CNS water homeostasis [11], and it is involved in such diverse functions as regulation of extracellular space volume, potassium buffering, cerebrospinal fluid circulation, interstitial fluid resorption, waste clearance, neuroinflammation, osmo-sensation, cell migration, and $\mathrm{Ca}^{2+}$ signaling [12]. However, AQP4 plays a different role in vasogenic and cytotoxic edema. During cerebral edema formation, the glial water channel AQP4 facilitates astrocyte swelling (i.e., cytotoxic swelling), and it is also responsible for the reabsorption of extracellular edema fluid (i.e. vasogenic edema) [1].

Yao et al [14] demonstrated that brain edema and cerebral infarct volume are reduced in AQP4-deficient mice after transient focal cerebral ischemia and reperfusion injury produced via 1-hour MCA occlusion followed by 23-h reperfusion. Hirt et al [15] found that the absence of AQP4 was associated with a decreased mortality and increased motor recovery 3-14 days after stroke. Studies have shown that AQP4 is a promising potential drug target for relieving cerebral edema caused by stroke [16], and the broad clinical utility of AQP4 modulators has been speculated. Some studies that have reported that pretreatment with TGN020, an AQP4 inhibitor, resulted in significant reduction in the severity of brain edema associated with brain ischemia in mice, based on 7.0-T magnetic resonance imaging (MRI) [17]. Moreover, TGN-020 [18] when administered after the onset of ischemia, significantly reduced edema, glial scar formation, albumin effusion, and apoptosis 3 and 7 days after MCAO. A number of studies that have demonstrated that pretreatment with atorvastatin decreased AQP4 expression via suppression of the p38MAPK pathway, which might be the mechanism by which atorvastatin alleviates ischemic cerebral edema [19].

Nakano et al [20] also observed that pretreatment with goreisan significantly decreased brain water content and AQP4 expression. Li et al [21] demonstrated that astragaloside IV significantly attenuated the cerebral water content and improved neurological outcomes during cerebral ischemia/reperfusion (I/R) injury, when compared with the vehicle group. This effect was correlated with downregulation of the expressions of matrix metalloproteinase-9 (MMP-9) and AQP4.

Moreover, the combined use of paeoniflorin and astragaloside IV was shown to reduce the incidence of ischemic brain edema via the downregulation of connexin 43, which was associated with downregulation of Cx43, resulting in a decrease in AQP4 [22]. The results obtained in the present study have also shown that AQP4 is the key link in the glial lymphatic system, a new clearance system in the brain which was identified in the rodent brain in 2012, and considered as a new anatomical route for CSF-interstitial fluid flow [23]. These results indicate that a clear decrease in AQP4 expression results in a decrease in encephaledema, and improvements in nerve function and histopathology, which might be the mechanisms through which SXDP provides the brain cells with resistance and/or tolerance against acute ischemic stroke. The effect of SXDP on AQP4-related upstream or downstream mechanisms in MCAO rats was not investigated in this study. This will be addressed in subsequent studies. Overall, it is apparent that SXDP provided favorable therapeutic effect on cerebral ischemia, at least partly via AQP4 suppression. This highlights the potential efficacy of oral consumption of SXDP in the prevention of stroke in patients who are at a high risk of recurrent stroke or transient ischemic attack.

\section{CONCLUSION}

These data indicate that SXDP pretreatment exerts neuroprotective effect against cerebral ischemia in rats through a mechanism involving the inhibition of the expression of AQP4. These results imply the therapeutic potential of AQP4 inhibitor for the management of cerebral stroke. 


\section{DECLARATIONS}

\section{Acknowledgement}

The work was supported by grants from the Chengdu University of Traditional Chinese Medicine (no. 030/030054111 and Sichuan Medical Association (no. Q16021). Special thanks to Professor Zhuyun Yan of Chengdu University of Traditional Chinese Medicine, College of Pharmacy.

\section{Conflict of interest}

The authors declare that there is no conflict of interest with regard to this study.

\section{Contribution of authors}

We declare that this work was done by the authors named in this article, and all liabilities pertaining to claims relating to the content of this article will be borne by the authors. Wei-yin Chen, Shuo-guo Jin and Dong-dong Yang conceived and designed the study. Li Zhang, Mei-jun Liu, Hong-hui Sun, and Ning-jing Ran collected and analyzed the data. Shuoguo Jin, Jili Deng, Fang Yang and Ze-ran Chen wrote the manuscript. Shuo-guo Jin and Ji-li Deng contributed equally to this work. All authors read and approved the manuscript for publication.

\section{Open Access}

This is an Open Access article that uses a funding model which does not charge readers or their institutions for access and distributed under the terms of the Creative Commons Attribution License (http://creativecommons.org/licenses/by/ 4.0) and the Budapest Open Access Initiative (http://www.budapestopenaccessinitiative.org/rea d), which permit unrestricted use, distribution, and reproduction in any medium, provided the original work is properly credited.

\section{REFERENCES}

1. Chen Z. The Third Nationwide Survey on Causes of Death. Beijing: The Peking Union Medical College Press; 2008

2. Wang $W Z$, Jiang $B$, Sun $H X, R u X J$, Sun $D L$, Wang $L H$, Wang $L M$, Jiang $Y, L i Y C$, Wang $Y L$, et al. Prevalence, Incidence, and Mortality of Stroke in China: Results from a Nationwide Population-Based Survey of 480687 Adults. J Circulation 2017; 8: 759-771.

3. Jin SG, Chen WY, Liu FY, Huang M, Zhu GX, Wang HM. Research on the impact of pretreatment of Shenxiong drop pill on the size of ischemic area and nissl bodies of rats model with middle cerebral artery occlusion. Chinese J Basic Med TCM 2009; 7: 501-503.

4. Jiang JY, Chen WY, Zhu B, Liu FY, Feng XM, Yu F. Impact of Shenxiong drop pill and cyclophosphamide on the expression of TNF- $\alpha$ mRNA of rats model with MACO. Huaxi Medical J 2008; 4: 810- 812.

5. Ma DC, Chen WY, Li HG, Liang XY, Zhang L, Zhao JW, Yang $F$. The impact of Shenxiong drop pill on brain nerve plasticity after cerebral ischemia-reperfusion. Chinese patent medicine 2012; 5: 814-818.

6. Jin SG, Liu FY, Liang JT, Yang DD, Chen WY. Impact of Shenxiong drop pill on t-PA and PAl-1 of plasma of rats with MACO. Hubei J Trad Chin Med 2014; 7: 19-20.

7. Cao P, Chen WY, Yang F, Zhu BD, Liu FY, Xie W, Fang $Y$. Impact of Shenxiong drop pill on the size of ischemic area and expression of NF-KB in the brain of rats model with MACO. J Sichuan University (Medical Edition) 2014; 5: 801-804.

8. Verkman AS, Smith AJ, Phuan PW, Tradtrantip L, Anderson MO. The aquaporin-4 water channel as a potential drug target in neurological disorders. Expert Opin Ther Targets 2017;21(12):1161-1170.

9. Longa EZ, Weinstein PR, Carlson S, Cummins $R$. Reversible middle cerebral artery occlusion without craniectomy in rats. J Stroke 1989; 20: 84 -91.

10. Bederson JB, Pitts LH, Tsuji M, Nishimura MC, Davis RL, Bartkowski H. Rat middle cerebral artery occlusion: evaluation of the model and development of a neurologic examination. Stroke 1986; 17(3):472-6.

11. Hubbard JA, Szu JI, Binder DK. The role of aquaporin-4 in synaptic plasticity, memory and disease. J Brain Res Bull 2017; 136(1): 118-129.

12. Nagelhus EA, Ottersen OP. Physiological roles of aquaporin-4 in brain. J Physiol Rev 2013; 93(4): 1543.

13. Jiao FZ, Gong ZJ. Research progression the correlation between aquapor4 and brain edema. Chin J Dific Compl Cas 2017; 16(02): 199-202+206.

14. Yao X, Derugin N, Manley GT, Verkman AS. Reduced brain edema and infarct volume in aquaporin-4 deficient mice after transient focal cerebral ischemia. Neurosci Lett 2015; 584: 368-372.

15. Hirt L, Fukuda AM, Ambadipudi K, Rashid F, Binder D, Verkman A, Ashwa S, Obenaus A, Badaut J. Improved long-term outcome after transient cerebral ischemia in aquaporin-4 knockout mice. J Cereb Blood Flow Metab 2017; 37(1): 277-290.

16. Verkman AS, Smith AJ, Phuan PW, Tradtrantip L, Anderson MO. The aquaporin-4 water channel as a potential drug target in neurological disorders. Expert Opin Ther Targets 2017; 21(12): 1161-1170.

17. Igarashi H, Huber VJ, Tsujita M, Nakada T. Pretreatment with a novel aquaporin 4 inhibitor, TGN-020, significantly reduces ischemic cerebral edema. Neurol Sci 2010; 32(1): 113-116.

18. Pirici I, Balsanu TA, Bogdan C, Margaritescu C, Divan T, Vitalie V, Mogoanta L, Pirici D, Carare RO, Muresanu DF. Inhibition of Aquaporin-4 Improves the Outcome of

Trop J Pharm Res, August 2020; 19(8): 1721 
Ischaemic Stroke and Modulates Brain Paravascular Drainage Pathways. Int J Mol Sci 2018; 19(1): 46.

19. Cheng ZJ, Dai TM, Shen YY, He JL, Li J, Tu JL. Atorvastatin Pretreatment Attenuates Ischemic Brain Edema by Suppressing Aquaporin 4. J Stroke Cerebrovascular Dis 2018; 27(11): 3247-3255.

20. Nakano $T$, Nishigami $C$, Irie $K$, Shigemori $Y$, Sano $K$, Yamashita $Y$, Myose $T$, Tominaga $K$, Matsuo $K$ Nakamura $Y$ et al. Goreisan Prevents Brain Edema after Cerebral Ischemic Stroke by Inhibiting Aquaporin 4 Upregulation in Mice. J Stroke Cerebrovasc Dis 2018; 27(3): 758-763
21. Li M, Ma RN, Li LH, Qu YZ, Gao GD. Astragaloside IV reduces cerebral edema post-ischemia/reperfusion correlating the suppression of MMP-9 and AQP4. Eur J Pharmacol 2013; 715(1-3): 189-195.

22. Chu H, Huang C, Gao Z, Dong J, Tang Y, Dong Q. Reduction of Ischemic Brain Edema by Combined use of Paeoniflorin and Astragaloside IV via DownRegulating Connexin 43. Phytother Res 2017; 31(9): 1410-1418.

23. Rasmussen MK, Mestre $H$, Nedergaard $M$. The glymphatic pathway in neurological disorders. Lancet Neurol 2018; 17(11): 1016-1024. 